\title{
Triple-region structure for turbulent flow in a square duct: A finite element approach
}

\author{
F.M. Wang*,1 B.C. Khoo, Y.T. Chew, K.S. Yeo \\ Department of Mechanical and Production Engineering, National University of Singapore, Kent Ridge, Singapore 0511, \\ Singapore
}

\begin{abstract}
The Reynolds-averaged Navier-Stokes equations for describing the turbulent flow in a straight square duct are formulated with two different turbulence models. The governing equations are then expanded as a multi-deck structure in a plane perpendicular to the streamwise direction, with each deck characterized by its dominant physical forces as commonly carried out in analytical work using triple-deck expansion. The resulting equations are numerically integrated using higher polynomial (H-P) finite element technique for each cross-sectional plane to be followed by finite difference representation in the streamwise direction until a fully developed state is reached. The computed results using the two different turbulence models show fair agreement with each other, and concur with the vast body of available experimental data. There is also general agreement between our results and the recent numerical works anisotropic $k-\varepsilon$ turbulence model.
\end{abstract}

\section{Introduction}

In many internal flows of engineering interest, ducts of non-circular cross-section are used extensively. Numerous examples exist like the flow in heat exchangers, turbomachineries, airconditioning systems and the air-intake ducts of the jet engines including the section leading to the propulsion unit. In some of these applications, the presence of the secondary flows in the non-circular plane perpendicular to the streamwise direction is used precisely to enhance mixing or turbulent transport. Although the magnitude of the secondary velocity is of the order of a few percent of the bulk streamwise flow, it has far reaching consequences in terms of turbulent heat/mass transport. For a square or rectangular section, the turbulence-induced secondary

\footnotetext{
* Corresponding author.

${ }^{1}$ Permanent address: Institute of Mechanics, Academia Sinica, Beijing 100080, China.
} 
motion causes distortion of the mean streamwise velocity towards the corner and a complex three-dimensional flow results.

Many experimental studies were carried out to investigate the resulting turbulent flow field of a straight non-circular duct. Ref. [1] can be credited with the first to observe the secondary motion of such flow using flow visualization. However, it was not until about another 30 years later that interest was revived in the said topic with the work by Hoagland [2]. Numerous other works follow, see e.g., [3-7]. Most of these studies were confined to flow in a square duct. Flow in other types of cross-sectional shapes can be found in [8] for a triangular section and [9] for a rectangular section. In the more complicated cases, surface roughness of the wall are included as a varying parameter as in [9] or the use of angled ribs as in [10].

Numerical computation of the secondary flow in straight non-circular duct was first carried out by Launder and Ying [6]. Since then, a number of investigators have also used the turbulence model (or extended variations of the model) as outlined by Launder and Ying for computation of the primary and secondary velocities of the said flow. Such works can be found in $[7,11,12]$. All these calculations adopted the strategy outlined by Patankar and Spalding [13] which is found in the algorithm called SIMPLE. A subsequent version is called SIMPLER.

In many engineering applications, there is a shaped variation of the non-circular duct in the streamwise direction, not to mention the even more complex scenario of directional variation. It is our intention in this paper to combine the use of finite element and finite difference techniques to numerically integrate the governing equations for flow in a straight square duct with the provision of facilitating future work where there is variation of both cross-sectional shape and direction in the streamwise direction. In our work, an order-or-magnitude analysis of the time-averaged governing equations gives rise to the parabolized Navier-Stokes equations. These resulting equations are described in a multi-region framework on a plane perpendicular to the streamwise direction thereby reflecting the local balance of the dominant physical forces in each region. Two different methods are then adopted for turbulence modelling. Starting from the algebraic Reynolds stress equation adopted from [14], one method involves solving the stress components using Chen and Patel's [15] two-layer approach for modelling of the eddy-viscosity relations. In this first way, the no-slip boundary condition at the wall is used in place of the common wall function approach which is used in the second method. for the latter case, the eddy viscosity based on $k-\varepsilon$ model is used throughout and is similar to the procedure outlined by Demuren and Rodi [14]. In the implementation, the numerical integration in the cross-plane is based on a finite element procedure with a bicubic Hermite polynomial function with 16 degrees of freedom and $C^{1}$ continuity at the element interfaces. (This ensures continuity of gradient across the boundaries of the multi-regions.) The finite element method would greatly facilitate future work when complex cross-sectional shape are implemented. A finite difference marching procedure is used for the streamwise direction thereby facilitating other future work where varying cross-sectional shape can be studied or for the scenario of changing flow direction with radius of curvature. The present results obtained for flow in straight square duct compare well with each other for the two types of turbulence modelling. Comparisons are also made with available experimental data and other numerical works, including the recent studies using anisotropic $K-\varepsilon$ turbulence model [16-18], showing good agreement. 


\section{Mathematical model}

\subsection{Reynolds-averaged Navier-Stokes equations}

The Reynolds-averaged Navier-Stokes equations governing the mean flow in a straight square duct (Fig. 1) can be written in Cartesian tensor notation as follows: the continuity equation

$$
\frac{\mathrm{d} U_{j}^{\prime}}{\mathrm{d} x_{j}^{\prime}}=0,
$$

the streamwise momentum equation

$$
U_{j}^{\prime} \partial U_{1}^{\prime} / \partial x_{j}^{\prime}=-(1 / \rho) \overline{\mathrm{d} P^{\prime} / d x_{1}^{\prime}}-\partial \overline{u_{1}^{\prime} u_{j}^{\prime}} / \partial x_{j}^{\prime}+v \partial^{2} U_{1}^{\prime} / \partial x_{j}^{\prime} \partial x_{j}^{\prime},
$$

the momentum equations for the velocities in the cross-plane, $U_{2}^{\prime}$ and $U_{3}^{\prime}$,

$$
U_{j}^{\prime} \partial U_{L}^{\prime} / \partial x_{j}^{\prime}=-(1 / \rho) \partial P^{\prime} / \partial x_{L}^{\prime}-\partial \overline{u_{L}^{\prime} u_{j}^{\prime}} / \partial x_{j}^{\prime}+v \partial^{2} U_{L}^{\prime} / \partial x_{j}^{\prime} \partial x_{j}^{\prime}, \quad L=2,3 .
$$

Here the superscript ' represents the dimensional quantity, $U_{j}^{\prime}$ is the component of the mean velocity vector in the $x_{j}$-direction $(j=1,2,3), P^{\prime}$ is the pressure field, $\rho$ is the density and $v$ is the kinematic viscosity. From order of magnitude analysis, the pressure gradient in the streamwise direction is replaced by the cross-sectional average pressure gradient $\overline{\mathrm{d} P^{\prime} / \mathrm{d} x_{1}^{\prime}}$.

Next, we select $U^{*}$ and $V^{*}$ as the streamwise and transverse velocity component scales, respectively, for purpose of non-dimensionalization. The corresponding length scales for this process are denoted by $L_{1}$ and $L_{2}$, while the turbulent characteristic length and energy scales are

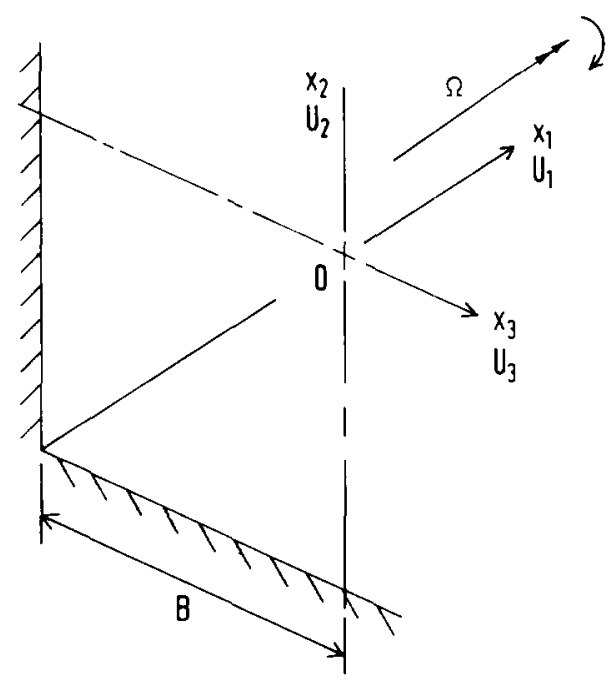

Fig. 1. Coordinate system and pertinent variables with 0 as the origin. 
denoted by $L^{\prime}$ and $V^{\prime 2}$, respectively. The various dimensionless variables are defined as follows:

$$
\begin{aligned}
& U_{1}=U_{1}^{\prime} / U^{*}, \quad U_{L}=U_{L}^{\prime} / V^{*}, \quad x_{1}=x_{1}^{\prime} / L_{1}, \quad x_{L}=x_{L}^{\prime} / L_{2}, \\
& u_{i, j}=\left(\overline{\left.u_{i}^{\prime} u_{j}^{\prime} / V^{\prime 2}\right) R_{i j}} \quad \bar{P}\left(x_{1}\right)+\delta P\left(x_{L}\right)=P^{\prime} / \rho U^{* 2} \quad(L=2,3),\right.
\end{aligned}
$$

where $R_{i, j}=\overline{u_{i}^{\prime} u_{j}^{\prime}}\left(\overline{u_{i}^{\prime} u_{i}^{\prime}} \overline{u_{j}^{\prime} u_{j}^{\prime}}\right)^{-0.5}$ is the correlation coefficient of turbulent velocities. Based on the experimental results that the secondary velocities are $\mathrm{O}(2-3 \%)$ of the streamwise bulk velocity and others, we set $\delta \equiv \mathrm{O}\left(1 / R^{0.5}\right)$ as a small parameter and the following relations:

$$
\mathrm{O}\left(V^{*} / U^{*}\right)=\mathrm{O}\left(L_{2} / L_{1}\right)=\mathrm{O}\left(V^{\prime 2} / U^{* 2}\right)=\delta, \quad R_{i j}=\mathrm{O}(1) .
$$

Here $R$ is taken to be Reynolds number, $U_{\mathrm{b}} D_{\mathrm{h}} / v$, based on the streamwise bulk velocity $U_{\mathrm{b}}\left(=U^{*}\right)$ and the hydraulic diameter $D_{\mathrm{h}}\left(=L_{2}\right)$ of the test section while $L_{1}$ is the length of the duct. In the subsequent implementation, the relations outlined in (5) are taken to be exact.

The dimensionless form of the governing equations are summarized below in the form.

$$
\begin{aligned}
& \frac{\partial U_{i}}{\partial x_{i}}=0 \\
& U_{i} \partial U_{1} / \partial x_{i}=-\overline{\mathrm{d} P / \mathrm{d} x_{1}}-\partial u_{1 L} / \partial x_{L}+(1 / R)\left(L_{1} / L_{2}\right) \partial^{2} U_{1} / \partial x_{L} \partial x_{L}, \\
& \delta U_{i} \partial U_{2} / \partial x_{i}=-\partial P / \partial x_{2}-\delta \partial u_{12} / \partial x_{1}-\partial u_{2 L} / \partial x_{L}+\delta(1 / R)\left(L_{1} / L_{2}\right) \partial^{2} U_{2} / \partial x_{L} \partial x_{L} \\
& \delta U_{i} \partial U_{3} / \partial x_{i}=-\partial P / \partial x_{3}-\delta \partial u_{13} / \partial x_{1}-\partial u_{3 L} / \partial x_{L}+\delta(1 / R)\left(L_{1} / L_{2}\right) \partial^{2} U_{3} / \partial x_{L} \partial x_{L},
\end{aligned}
$$

where $i=1,2,3$, and $L=2,3$.

\subsection{Turbulence model}

The modelled transport equation derived by Demuren and Rodi [14] can be written as

$$
\begin{aligned}
& (1-\alpha)\left[-\overline{u_{i}^{\prime} u_{L}^{\prime}} \partial U_{j}^{\prime} / \partial x_{L}^{\prime}-\overline{u_{j}^{\prime} u_{L}^{\prime}} \partial U_{i}^{\prime} / \partial x_{L}^{\prime}\right]+\beta\left[\overline{u_{i}^{\prime} u_{L}^{\prime}} \partial U_{L}^{\prime} / \partial x_{j}^{\prime}+\overline{u_{j}^{\prime} u_{L}^{\prime}} \partial U_{L}^{\prime} / \partial x_{i}^{\prime}\right] \\
& \quad-\gamma k^{\prime}\left[\partial U_{i}^{\prime} / \partial x_{j}^{\prime}+\partial U_{j}^{\prime} / \partial x_{i}^{\prime}\right]-\left(\varepsilon^{\prime} / k^{\prime}\right)\left[C_{1} u_{i}^{\prime} u_{j}^{\prime}+\frac{2}{3}\left(1-\alpha-\beta-C_{1}\right) \delta_{i j} k^{\prime}\right]=0,
\end{aligned}
$$

where the various symbols take on usual meanings and the empirical parameters are given as

$$
C_{1}=1.5, \quad \alpha=0.7637, \quad \beta=0.1091, \quad \gamma=0.182 .
$$

Following Demuren and Rodi, the Reynolds stress components $\overline{u_{i}^{\prime} u_{j}^{\prime}}$ are solved from Eq. (10) with the adoption of an eddy-viscosity relation. From here, two methods will be used for expressing the eddy-viscosity relation.

In the first method, we use the two-layer turbulence model of Chen and Patel [15]. For the region far away from the wall, the usual $k-\varepsilon$ turbulence model is used where the turbulent eddy viscosity $\mu_{t}^{\prime}$ is related to the kinetic energy $k^{\prime}$ and its rate of dissipation $\varepsilon^{\prime}$ by the relation

$$
\mu_{t}^{\prime}=C_{\mu} k^{\prime 2} / \varepsilon^{\prime} \text {. }
$$


$k^{\prime}$ and $\varepsilon^{\prime}$ are evaluated from the following equations:

$$
\begin{aligned}
& U_{j}^{\prime} \partial k^{\prime} / \partial x_{j}^{\prime}=\partial\left[\left(v+\mu_{t}^{\prime} / \sigma_{k}\right) \partial k^{\prime} / \partial x_{j}^{\prime}\right] / \partial x_{j}^{\prime}+P_{k}^{\prime}-\varepsilon^{\prime}, \\
& U_{j}^{\prime} \partial \varepsilon^{\prime} / \partial x_{j}^{\prime}=\partial\left[\left(v+\mu_{t}^{\prime} / \sigma_{\varepsilon}\right) \partial \varepsilon^{\prime} / \partial x_{j}^{\prime}\right] / \partial x_{j}^{\prime}+\left(\varepsilon^{\prime} / k^{\prime}\right)\left(C_{\varepsilon 1} P_{k}^{\prime}-C_{\varepsilon 2} \varepsilon^{\prime}\right),
\end{aligned}
$$

where $i, j, k=1,2,3$, and $C_{\mu}, \sigma_{k}, \sigma_{\varepsilon}, C_{\varepsilon 1}, C_{\varepsilon 2}$ are empirical constants given by the following standard values

$$
C_{\mu}=0.09, \quad \sigma_{k}=1.0, \quad \sigma_{\varepsilon}=1.3, \quad C_{\varepsilon 1}=1.44, \quad C_{\varepsilon 2}=1.92 .
$$

In Eqs. (13) and (14), $P_{k}^{\prime} \equiv-\overline{u_{i}^{\prime} u_{k}^{\prime}} \partial U_{i}^{\prime} / \partial x_{k}^{\prime}$ represents the production of the turbulent kinetic energy.

Similar to [15], for the region near to the wall, $\mu_{t}^{\prime}$ and $\varepsilon^{\prime}$ are calculated from the expressions:

$$
\mu_{t}^{\prime}=C_{\mu} k^{0.5} l_{\mu}^{\prime}, \quad \varepsilon^{\prime}=k^{1.5} / l_{\varepsilon}^{\prime} .
$$

Here $l_{\mu}^{\prime}=C_{L} y\left[1-\exp \left(-R_{y}^{\prime} / 70\right)\right], l_{\varepsilon}^{\prime}=C_{L} y\left[1-\exp \left(R_{y}^{\prime} / 2 C_{L}\right)\right], C_{L}=k C_{\mu}^{-0.75}, R_{y}^{\prime}=R k^{0.5} y$ and $k$ is the von Karman constant of 0.418 . For the above, $y$ is defined as the distance away from the wall. In the corner region, $y$ is taken to be the minimum of the two distances perpendicular from the wall. The turbulent kinetic energy is calculated from Eq. (13).

For non-dimensionalization purpose, we define the variables $k$ and $\varepsilon$ as

$$
k=k^{\prime} / V^{\prime 2}, \quad \varepsilon=\varepsilon^{\prime} L^{\prime} / V^{\prime 3},
$$

From previous experimental results, we establish that $\mathrm{O}\left(u_{i j}\right) \simeq \mathrm{O}(\delta), \quad \mathrm{O}\left(C_{\mu} / \varepsilon\right) \simeq$ $\mathrm{O}\left(C_{\mu} \beta / C_{1} \varepsilon\right) \simeq \mathrm{O}(1)$ and $\mathrm{O}\left(L^{\prime} / L_{2}\right) \simeq \mathrm{O}\left(V^{\prime} / U^{*}\right) \simeq \mathrm{O}\left(\delta^{0.5}\right)$, and the six components of Reynolds stress tensor can be evaluated from Eq. (10) as

$$
\begin{aligned}
& u_{11}=\left(2 /\left(3 C_{1}\right)\right)\left(\alpha+\beta+C_{1}-1\right) k-2(1-\alpha)\left(\mu_{t} / C_{1}\right)(k / \varepsilon)\left[\left(\partial U_{1} / \partial x_{2}\right)^{2}+\left(\partial U_{1} / \partial x_{3}\right)^{2}\right], \\
& u_{22}=\left(2 /\left(3 C_{1}\right)\right)\left(\alpha+\beta+C_{1}-1\right) k-2 \beta\left(\mu_{t} / C_{1}\right)(k / \varepsilon)\left(\partial U_{1} / \partial x_{2}\right)^{2}-2 \delta \mu_{t}\left(\partial U_{2} / \partial x_{2}\right), \\
& u_{33}=\left(2 /\left(3 C_{1}\right)\right)\left(\alpha+\beta+C_{1}-1\right) k-2 \beta\left(\mu_{t} / C_{1}\right)(k / \varepsilon)\left(\partial U_{1} / \partial x_{3}\right)^{2}-2 \delta \mu_{t}\left(\partial U_{3} / \partial x_{3}\right), \\
& u_{12}=-\mu_{t}\left(\partial U_{1} / \partial x_{2}\right), \\
& u_{13}=-\mu_{t}\left(\partial U_{1} / \partial x_{3}\right), \\
& u_{23}=-2 \beta\left(\mu_{t} / C_{1}\right)(k / \varepsilon)\left[\left(\partial U_{1} / \partial x_{2}\right)\left(\partial U_{1} / \partial x_{3}\right)\right]-2 \delta \mu_{t}\left[\partial U_{2} / \partial x_{3}+\partial U_{3} / \partial x_{2}\right] .
\end{aligned}
$$

The turbulent viscosity $\mu_{t}$ for application to the region away and near to the wall are expressed as

$$
\mu_{t}=C_{\mu} k^{2} / \varepsilon
$$

and

$$
\mu_{t}=C_{\mu} k^{0.5} l_{\mu},
$$

respectively. $k$ is evaluated from the equation

$$
U_{j} \partial k / \partial x_{j}=\left(L_{1} / L_{2}\right) \partial\left(\left[(1 / R)+\left(C_{\mu} / \sigma_{k}\right)\left(k^{2} / \varepsilon\right)\right] \partial k / \partial x_{L}\right) / \partial x_{L}+\left(L_{1} / L_{2}\right)\left(P_{0}-\varepsilon\right),
$$


while the dissipation term $\varepsilon$ is governed by the expression

$$
\begin{aligned}
U_{j} \partial \varepsilon / \partial x_{j}= & \left(L_{1} / L_{2}\right) \partial\left(\left[(1 / R)+\left(C_{\mu} / \sigma_{\varepsilon}\right)\left(k^{2} / \varepsilon\right)\right] \partial \varepsilon / \partial x_{L}\right) / \partial x_{L} \\
& +\left(L_{1} / L_{2}\right)(\varepsilon / k)\left(C_{\varepsilon 1} P_{0}-C_{\varepsilon 2} \varepsilon\right)
\end{aligned}
$$

for the region far away from the walls. In the near-wall regions, we take

$$
\varepsilon=k^{1.5} / l_{\varepsilon} \text {. }
$$

For the above Eqs. (24) and (28),

$$
P_{0}=-u_{1 L} \partial U_{L} / \partial x_{L}-\delta\left(u_{11} \partial u_{1} / \partial x_{1}+u_{j L} \partial \mathrm{U}_{j} / \partial x_{L}\right) \quad \text { for } j, L=2,3
$$

and $l_{\mu}=C_{L} x_{L}\left[1-\exp \left(-R_{x} / 70\right)\right], l_{\varepsilon}=C_{L} x_{L}\left[1-\exp \left(-R_{x} / 2 C_{L}\right)\right], R_{x}=R k^{0.5} x_{L}, C_{L}=k C_{\mu}^{-0.75}$.

In the second turbulence model, the modelled transport equation as given by (10) is the same except that the empirical parameters are made dependent on the distance from the walls. They are given as

$$
C_{1}=1.5+0.5 f, \quad \alpha=0.7637-0.06 f, \quad \beta=0.1091+0.06 f, \quad \gamma=0.182,
$$

where $f$ is the wall damping function. A linear relationship suggested by Lander et al. [19] is adopted and is written as follows:

$$
f=L_{L} /[y], \quad \text { where } 1 /[\bar{y}]=(2 / \pi) \int_{0}^{2 \pi}(1 / y) \Phi .
$$

Here $L_{L}$ is the macro length scale of the turbulent motion at the location considered, $y$ is the distance from the wall and $[\bar{y}]$ can be regarded as the average distance from the surrounding walls. $L_{L}$ is determined from the expression,

$$
L_{L}=\left(C_{\mu}^{0.75} / \underline{k}\right) k^{1.5} / \varepsilon^{\prime},
$$

where $k$ is the von Karman constant of 0.418 . The quantities $k^{\prime}$ and $\varepsilon^{\prime}$ can be evaluated from Eqs. (13) and (14), respectively, with $\mu_{t}^{\prime}=C_{\mu} k^{\prime 2} / \varepsilon^{\prime}$ as given in Eq. (12). In dimensionless form, solutions of $k$ and $\varepsilon$ are obtained from Eqs. (26) and (27), respectively, with $\mu_{t}=C_{\mu} k^{2} / \varepsilon$ applicable throughout the domain. The Reynolds stress terms are essentially of the same form as given above from Eqs. (18)-(23). Overall, the second turbulence model used here can be considered as the application of the isotropic $k-\varepsilon$ turbulence model up to a region close to the wall where the boundary conditions are specified by the wall functions.

\subsection{A vector-potential function formulation}

Following [13], the parabolic properties of the governing equations enable us to use the special vector-potential function formulation. In it, only the streamwise component of vorticity tensor needs to be taken into consideration. We can define the velocity vector as

$$
U=U_{1} I+U_{2} J+U_{3} K \equiv U_{1} I+V_{S},
$$


where $(\boldsymbol{I}, \boldsymbol{J}, \boldsymbol{K})$ are the unit vectors along the coordinates $\left(x_{1}, x_{2}, x_{3}\right)$. The vector $\boldsymbol{V}_{S}=\boldsymbol{I}_{\boldsymbol{n}}+\boldsymbol{S}_{0}$ is the velocity components in the transverse plane which is further decomposed into its irrotational $\left(\boldsymbol{I}_{n}\right)$ and solenoidal component $\left(\boldsymbol{S}_{0}\right) . \boldsymbol{I}_{n}$ can also be defined by a potential scalar function $\phi$ such that $\boldsymbol{I}_{n}=\operatorname{Grad} \phi$ while $\boldsymbol{S}_{0}$ can be made related to another scalar $\psi$ (regarded as a potential stream function) by the divergence free conditions of $\boldsymbol{S}_{0}$. In other words, $S_{02}=\partial \psi / \partial x_{3}$ and $S_{03}=-\partial \psi / \partial x_{2}$ for the $\boldsymbol{J}$ and $\boldsymbol{K}$ directions, respectively. Next, by taking the divergence of Eq. (33), $\phi$ is found to satisfy

$$
\partial^{2} \phi / \partial x_{2} \partial x_{2}+\partial^{2} \phi / \partial x_{3} \partial x_{3}=-\partial U_{1} / \partial x_{1}
$$

and the streamwise component of vorticity $(\Omega)$ is related to $\psi$ through

$$
\partial^{2} \psi / \partial x_{2} \partial x_{2}+\partial^{2} \psi / \partial x_{3} \partial x_{3}=\Omega=\partial U_{2} / \partial x_{3}-\partial U_{3} / \partial x_{2} .
$$

With the above formulation, the momentum equations in the transverse plane(Eqs. 8) and (9)) can be reduced to the form

$$
\begin{aligned}
\delta U_{i} \partial \Omega / \partial x_{i}= & \delta\left[\Omega \partial U_{1} / \partial x_{1}-\left(\partial U_{2} / \partial x_{1}\right)\left(\partial U_{1} / \partial x_{3}\right)+\left(\partial U_{3} / \partial x_{1}\right)\left(\partial U_{1} / \partial x_{2}\right)\right. \\
& \left.+\partial^{2} u_{13} / \partial x_{1} \partial x_{2}-\partial^{2} u_{12} / \partial x_{1} \partial x_{3}\right]+\partial^{2}\left(u_{33}-u_{22}\right) / \partial x_{2} \partial x_{3} \\
& -\partial^{2} u_{23} / \partial x_{3} \partial x_{3}+\partial^{2} u_{23} / \partial x_{2} \partial x_{2}+(\delta / R)\left(L_{1} / L_{2}\right)\left(\partial^{2} \Omega / \partial x_{L} \partial x_{L}\right) .
\end{aligned}
$$

Here $i=1,2,3$, and $L=2,3, U_{2}=\partial \phi / \partial x_{2}+\partial \psi / \partial x_{3}$ and $U_{3}=\partial \phi / \partial x_{3}-\partial \psi / \partial x_{2}$.

\subsection{Multiple structure}

In both channel and boundary layer flows, the effects of kinematic viscosity and turbulence stresses ensure that there is both convection and diffusion of turbulence quantities in the streamwise and transverse directions, respectively. Because of this interaction, a multi-deck structure exists whereby each deck is characterized by a balance of its most dominant physical forces. The use of multi-region framework for the description of our turbulent flow is very similar to that carried out in analytical work using triple-deck expansion for better elucidation of the flow physics.

From past literature [20], the turbulent boundary layer flows can be roughly divided into regions. Next to the wall is the viscous sublayer $\left(0 \leqslant y \leqslant 5 v / U_{\tau}\right)$, followed by the interim layer $\left(5 v / U_{\tau} \leqslant y \leqslant 30 v / U_{\tau}\right)$, the logarithmic layer $\left(30 v / U_{\tau} \leqslant y \leqslant 0.2 \delta^{*}\right)$ and finally the outer region $\left(0.2 \delta^{*} \leqslant y\right)$. Here $U_{\tau}$ is the friction velocity and $\delta^{*}$ is the boundary layer thickness.

For our flow, we can restrict our analysis to a quarter of a square duct for reason of symmetry (see Figs. 1 and 2). We subdivide the flow into four regions: the viscos sublayer, the logarithmic layers (including the interim layers), the corner flow and a center region.

In the viscous sublayer, the effect of kinematic viscosity takes precedence and the turbulent eddy viscosity may be taken as zero. For the Chen and Patel turbulence model used, this region is computed as a laminar flow with the imposition of the non-slip boundary conditions at the wall. In the second model used, the boundary conditions are specified at locations outside this layer using the wall functions. 


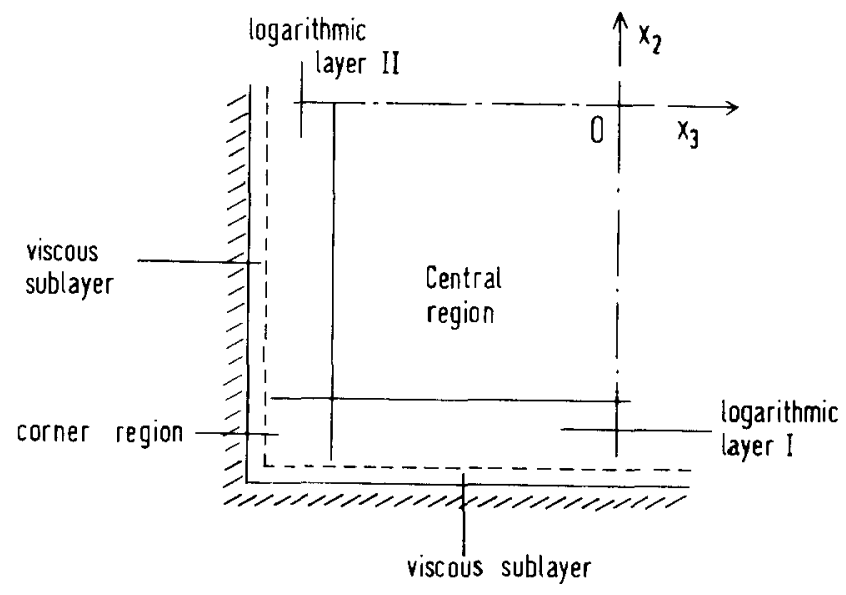

Fig. 2. Multi-region in a quarter square duct section.

For flow in the center region where the effect of turbulence is the most dominant, the local flow may be regarded as essentially potential where the damping terms association with viscous action in Eqs. (17) and (36) are neglected.

The flow in the logarithmic layers (refer to logarithmic layer I and II as in Fig. 2) is characterized by the complex interaction between wall shear stress and the turbulent kinetic stress. In it, we shall assume that the production of local kinetic energy is equal to the rate of viscous dissipation. Similar to the analytical work of triple-deck expansion in boundary layer flow, we shall employ coordinate transformation for this region. For example, in logarithmic layer I, we let

$$
x_{2}=-1+\delta \eta, \quad \text { where } \eta=\mathrm{O}(1)
$$

so that the quantities can be expanded as follows:

$$
\begin{aligned}
& U_{1}=U_{10}+\delta U_{11}+\cdots, \quad \Omega=\Omega_{0} / \delta+\Omega_{1}+\cdots, \\
& \phi=\delta^{2} \phi_{0}+\cdots, \quad \psi=\delta \psi_{0}+\delta^{2} \psi_{1}+\cdots, \\
& u_{m n}=\delta u_{m n o}+\delta^{2} u_{m n 1}+\cdots \quad \text { for the pair } m n=12,13,23, \\
& u_{m n}=u_{m n o}+\delta u_{m n 1}+\cdots \quad \text { for the pair } m n=22,23 .
\end{aligned}
$$

These quantities are for use in the governing equations where terms of similar order are collected for integration.

In the corner region, the flow appears, to be the result of a delicate balance between turbulence dissipation and induced secondary motion. For this reason, the expansion of the scaled transformation in the governing equations is carried out to a higher order. The transformations are given as

$$
x_{2}=-1+\delta \eta, \quad \eta=\mathrm{O}(1) ; \quad x_{3}=-1+\delta \xi, \quad \xi=\mathrm{O}(1),
$$


hence

$$
\begin{aligned}
& U_{1}=U_{10}+\delta U_{11}+\cdots, \quad \Omega=\Omega_{n}+\delta \Omega_{1}+\cdots, \\
& \phi=\delta \phi_{0}+\cdots, \quad \psi=\delta \psi_{0}+\cdots, \\
& u_{m n}=\delta u_{m n o}+\delta^{2} u_{m n 1}+\cdots \quad \text { for the pair } m n=12,13,22,23,33 .
\end{aligned}
$$

Again, terms of similar magnitude in the governing equations are collected for numerical integration.

As shown subsequently, the use of higher polynomial (H-P) finite element method for numerical integration in the cross-sectional plane implies $C^{1}$ continuity at each node and ensures smoothness of solution and its higher-order derivatives. This is very critical since the solutions associated with the governing equations of different regions require matching at the interfaces.

\section{Numerical implementation}

The governing equations derived in Section 2 can be summarized as four equations of parabolic type (Eqs. (7), (36), (26), and (27)) and two of Poisson type ((34) and (35)). The former is for the dependent variables set $Q_{i}=\left(U_{1}, \Omega, k, \varepsilon\right)$ and the latter is given as $\Psi_{i}=(\phi, \psi)$. Generally, the governing equations can be reduced to the form

$$
\begin{aligned}
& U_{1} \partial Q_{i} / \partial x_{1}+U_{L} \partial Q_{i} / \partial x_{L}=\partial\left[\Gamma_{L} \partial Q_{i} / \partial x_{L}\right] / \partial x_{L}+S_{i} \quad Q_{i} \in D, \\
& L\left(Q_{i}\right)=q Q_{i}+r \partial Q_{i} / \partial x_{L} \cdot n+s=0 \quad Q_{i} \in \partial D, \\
& \partial^{2} \Psi_{i} / \partial x_{L} \partial x_{L}=f_{i} \quad \Psi_{i} \in \mathbb{R}^{2},
\end{aligned}
$$

where $L=2,3$. Here $S_{i}$ expresses the corresponding remaining terms in Eqs. (7), (36), (26) and (27), $f_{i}$ denotes the right-hand side terms of Eqs. (34) and (35), $q, r, s$ and $\Gamma_{L}$ are specified coefficients, and $\boldsymbol{n}$ is the outward unit normal vector The solution domain is $D=\mathbb{R}^{2} \times\left[x_{1}(0), x_{1}\right]$ where $\mathbb{R}$ denotes the real number space. Its associated boundary is given by $\partial D=\partial \mathbb{R}^{2} \times\left[x_{1}(0), x_{1}\right]$.

For numerical integration of the governing equations, a finite-element approach is used for the cross-sectional plane. The finite-element domain is $D_{e}=\mathbb{R}_{e}^{2} \times x_{1}$. with $\mathbb{R}_{e}^{2}$ as the discretization of $\mathbb{R}^{2}$. The finite-element approximation of $Q_{i}^{h}$ on $D$ is then defined as

$$
Q_{i}^{h}\left(x_{L}, x_{1}\right)=\sum_{e=1}^{M} Q_{i}^{e}\left(x_{L}, x_{1}\right)=\sum_{e=1}^{M} \sum_{k=1}^{N} N_{k}\left(x_{L}\right)^{\mathrm{T}} Q_{i k}^{e}\left(x_{1}\right) .
$$

Here $Q_{i}^{h} \in H_{0}^{1}(D)$, where $H_{0}^{1}(D)$ denotes the Hilbert space of all functions possessing square integrable first derivative and satisfying the boundary constraint as given in (42). The superscript $e$ denotes the $e$ th finite element of the domain $D_{e}$ while the superscript $\mathrm{T}$ denotes the transpose of the corresponding matrix. The elements of the row matrix $N_{k}\left(x_{L}\right)^{\mathrm{T}}$ are polynomials on $x_{L}(L=2,3)$ constructed to form a cardinal basis.

The Galerkin finite element approach is to find $Q_{i}^{h} \in H_{0}^{1}(D)$ which satisfies the governing Equation (41).

$$
a\left(Q_{i}^{h}, U_{1}^{h}, N_{k}\right)+b\left(Q_{i}^{h}, U_{L}^{h}, N_{k}\right)=c\left(Q_{i}^{h}, N_{k}\right)+d\left(Q_{i}^{h}, N_{k}\right) \quad \forall N_{k} \in H_{0}^{1}(D),
$$


where

$$
\begin{aligned}
& a\left(Q_{i}^{h}, U_{1}^{h}, N_{k}\right)=\int_{D} N_{k} U_{1} \partial Q_{i}^{h} / \partial x_{1} \mathrm{~d} x_{L}, \\
& b\left(Q_{i}^{h}, U_{L}^{h}, N_{k}\right)=\int_{D} N_{k} U_{L}^{h} \partial Q_{i}^{h} / \partial x_{L} \mathrm{~d} x_{L}, \\
& c\left(Q_{i}^{h}, N_{k}\right)=\int_{D} \Gamma_{L}\left(\partial N_{k} / \partial x_{L}\right)\left(\partial Q_{i}^{h} / \partial x_{L}\right)+N_{k}\left[S_{p} Q_{i}^{h}+S_{p}\right] \mathrm{d} x_{L}, \\
& d\left(Q_{i}^{h}, N_{k}\right)=\oint_{\partial D} N_{k} L\left(Q_{i}^{h}\right) \mathrm{d} \sigma
\end{aligned}
$$

and $\mathrm{d} \sigma$ is the line integral. In the implementation, rectangular element utilizing the bicubic Hermite polynomials which provides 16 degrees of freedom and $C^{1}$ continuity is used. The elemental approximation to the dependent variables $Q_{i}$ follow

$$
Q_{i}^{e}\left(x_{L}, x_{1}\right)=\sum_{k=1}^{16} N_{k}\left(x_{L}\right) Q_{i k}^{e}\left(x_{1}\right)=\bar{F} B \bar{G}^{\mathrm{T}} .
$$

Here $B$ expresses the values of elemental solution and some of its derivative at the common nodes. Sometimes, it is called the information matrix and is given as

$$
B=\left[\begin{array}{cccc}
b_{1}\left(x_{1}\right) & b_{3}\left(x_{1}\right) & \frac{-h_{3 e}}{2} \frac{\partial}{\partial x_{3}} b_{1}\left(x_{1}\right) & \frac{h_{3 e}}{2} \frac{\partial}{\partial x_{3}} b_{3}\left(x_{1}\right) \\
b_{2}\left(x_{1}\right) & b_{4}\left(x_{1}\right) & \frac{-h_{3 e}}{2} \frac{\partial}{\partial x_{3}} b_{2}\left(x_{1}\right) & \frac{h_{3 e}}{2} \frac{\partial}{\partial x_{3}} b_{4}\left(x_{1}\right) \\
\frac{h_{2 e}}{2} \frac{\partial}{\partial x_{2}} b_{1}\left(x_{1}\right) & \frac{h_{2 e}}{2} \frac{\partial}{\partial x_{2}} b_{3}\left(x_{1}\right) & \frac{h_{2 e} h_{3 e}}{4} \frac{\partial^{2}}{\partial x_{2} \partial x_{3}} b_{1}\left(x_{1}\right) & \frac{h_{2 e} h_{3 e}}{4} \frac{\partial^{2}}{\partial x_{2} \partial x_{3}} b_{3}\left(x_{1}\right) \\
\frac{h_{2 e}}{2} \frac{\partial}{\partial x_{2}} b_{2}\left(x_{1}\right) & \frac{h_{2 e}}{2} \frac{\partial}{\partial x_{2}} b_{4}\left(x_{1}\right) & \frac{h_{2 e} h_{3 e}}{4} \frac{\partial^{2}}{\partial x_{2} \partial x_{3}} b_{2}\left(x_{1}\right) & \frac{h_{2 e} h_{3 e}}{4} \frac{\partial^{2}}{\partial x_{2} \partial x_{3}} b_{4}\left(x_{1}\right)
\end{array}\right]
$$

where $b_{i}(i=1,2,3,4)$ are the values of elemental solution at node $i$. and $h_{2 e}$ and $h_{3 e}$ are the respective lengths of the rectangular element in the $x_{2}$ and $x_{3}$ directions. $\bar{G}$ and $\bar{F}$ are two vector functions dependent on the local coordinates $\zeta$ and $\xi$, respectively. For our rectangular-shaped element, $\zeta$ and $\xi$ are functions of $x_{2}$ and $x_{3}$. (This approach can be easily extended to different finite element shapes to match the boundary contour of complex cross-sectional shapes via specification of the appropriate local coordinate system.)

In a similar way, the solution to the Poisson-type equation (43) can be written as

$$
\Psi_{i}\left(x_{L}\right) \cong \Psi_{i}^{h}\left(x_{L}\right) \equiv \sum_{e=1}^{M} \sum_{k=1}^{N=16} N_{k}\left(x_{L}\right)^{\mathrm{T}} \Psi_{i k}^{e}\left(x_{L}\right)
$$


and the finite approximation to the governing equation takes the form

$$
\int\left(\partial N_{k} / \partial x_{L}\right)\left(\partial \Psi_{i}^{h} / \partial x_{L}\right) \mathrm{d} x_{L}=\int N_{k} f_{i}^{h} \mathrm{~d} x_{L}+\oint N_{k} L\left(\Psi_{i}^{h}\right) \mathrm{d} \sigma,
$$

where $L$ is the representation for the boundary conditions. With $\Psi_{i}^{h}$ evaluated, the quantities for $U_{L}^{h}$ $(L=2,3)$ are obtained from

$$
U_{2}^{h}=\partial \phi^{h} / \partial x_{2}+\partial \psi^{h} / \partial x_{3} \text { and } U_{3}^{h}=\partial \phi^{h} / \partial x_{3}-\partial \psi^{h} / \partial x_{2} .
$$

Subsequently, the Reynolds stresses $u_{i j}$ are determined from the algebraic relations (18)-(23)

For numerical integration in the streamwise direction, an efficient forward marching finite difference procedure is adopted. In the present study, a streamwise step equivalent to 0.01 of the hydraulic diameter $D_{h}$ is used. The flow is computed with 100 elements in a quarter square duct cross-section. Calculations were also performed with 64 and 81 elements and the results agreed fairly well and consistently with each other.

For initial conditions, the steady-state laminar flow results for the streamwise velocity were used. The quantities $\Omega, \phi, \Psi$ were set equal to zero while the turbulent quantities $k$ and $\varepsilon$ assumed those values established using the standard wall function approach.

Boundary conditions are required at the walls and the plane of symmetry. Along the plane of symmetry, the velocity components normal to the surface were taken to be zero. The normal gradients to the same surface for the turbulent quantities $k$ and $\varepsilon$ took on null values. It then follows that the axial vorticity, the normal gradient of $\phi$ and the tangential gradient of $\psi$ had to be set equal to zero on this surface. Along the solids walls, $t w o$ sets of boundary conditions are required for the two different turbulence models adopted for our computation. In the first model using Chen and Patel's [15] two-layer approach, the velocity components and Reynolds stresses are set equal to zero, while the normal gradient of $\phi$ and the tangential gradient of $\psi$ assume null values. The axial vorticity is computed from (35). In the second case where the isotropic $k-\varepsilon$ turbulence model is applied up to the region close to the wall, the wall function approach is used. Essentially, the boundary condition for $U_{i}, k$ and $\varepsilon$ are specified at a location just outside the viscous sublayer where the logarithmic law of the wall prevails. Details of the implementation are given in [14]. The normal gradient of $\phi$ and the tangential gradient of $\psi$ to the walls are taken to be zero, and $\Omega$ is calculated from (35).

Computation of the flow in a square duct was carried out for three different $R$ of 50000,65000 and 250000 . Because our calculation started from the imposed initial conditions which does not simulate the physical entrance flow, the computations was first carried out to location $x_{1}^{\prime} / D_{h}=5$ solving only for $U_{1}, k$ and $\varepsilon$ using Boussinesq approximation. Further downstream, the full governing equations were integrated for all the dependent quantities until a fully developed state was reached. In our calculations, the fully developed condition occurred at $x_{1}^{\prime} / D_{h} \geqslant 80$.

\section{Results and discussion}

As mentioned above, our flow computations were carried out using two different turbulence models, namely, the two-layer model of Chen and Patel [15] and the isotropic turbulence model as 
used in [14]. In our discussion, we shall henceforth refer to the former as turbulence model I (TM I) and the latter as turbulence model II (TM II). Shown in Fig. 3 are the contours of the fully developed streamwise velocity distribution evaluated at $R=50000$ for TM I and TM II for comparison to the experimental results of Melling and Whitelaw [21] at $R=42000$ and the numerical computation by Nisizima [18] at $R=40860$. The results of TM I and TM II agree well with each other except for the central region where there is a slightly greater variation. The implication is that the primary velocity distribution is not too unduly affected by the use of either turbulence models. It gives us assurance in the use of the physical non-slip boundary condition (TM I), which can be easily generalize to other types of flows, as opposed to the need to specify the wall functions TM II), which is partly dependent on available experimental data. On the whole, both sets of data concur fairly well with the experimental results. However, there are some variations on the velocity contours when compared to the numerical prediction by Nisizima, who used an anisotropic $k-\varepsilon$ turbulence model. The latter has comparatively larger contour quantities at similar location on the cross-sectional plane. Nisizima has attributed the differences to the less fully developed flow condition prevailing for Melling and Whitelaw's experimental data obtained at $x_{1}^{\prime} / D_{h}=36.8$. In our subsequent Fig. 4 showing the evolution of the stream function at various streamwise locations, it is found that there is relatively little variation in the stream function contour at location $x_{1}^{\prime} / D_{h}=42$ and 84 , thereby suggesting that the results of Melling and Whitelaw may be asymptotically close to the fully developed state.

Fig. 4 shows the evolution of the secondary flow by depicting the stream function $(\psi)$ contours at various downstream location of $x_{1}^{\prime} / D_{h}=25,42$ and 84 . The results are obtained for $R=50000$. It should be noted that these plots are only intended to show generally how the flow features evolve under the influence of the turbulent stresses and is not for strict comparison to the experimental

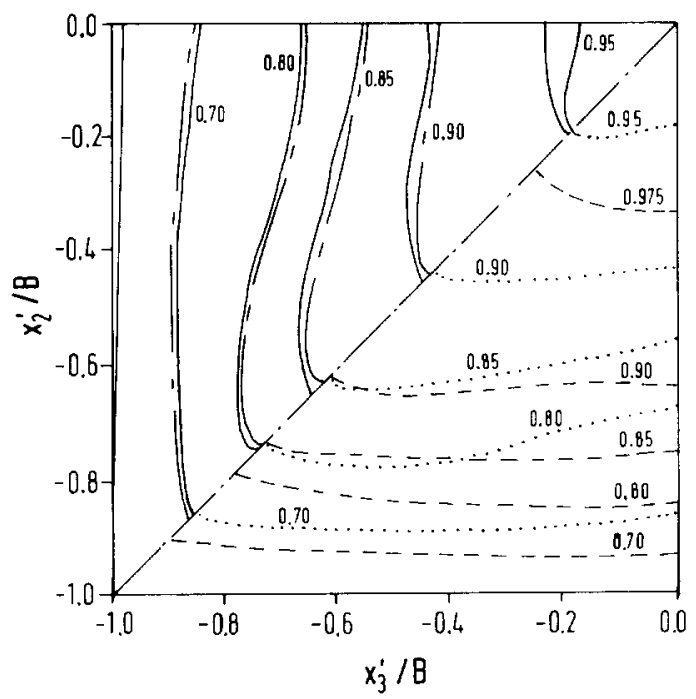

Fig 3. Contours of primary streamwise velocity $\left(U_{1}^{\prime} / U_{1 \max }^{\prime}\right)$. Present computation at $R=50000:(-\ldots \ldots--)$ TM I and ( $\ldots$ ) TM II; $(\ldots--)$ computations by Nisizima [18] at $R=40806 ;(\ldots \ldots \ldots)$ experimental results by Melling and Whitelaw [21] at $R=42000$. 
(a)

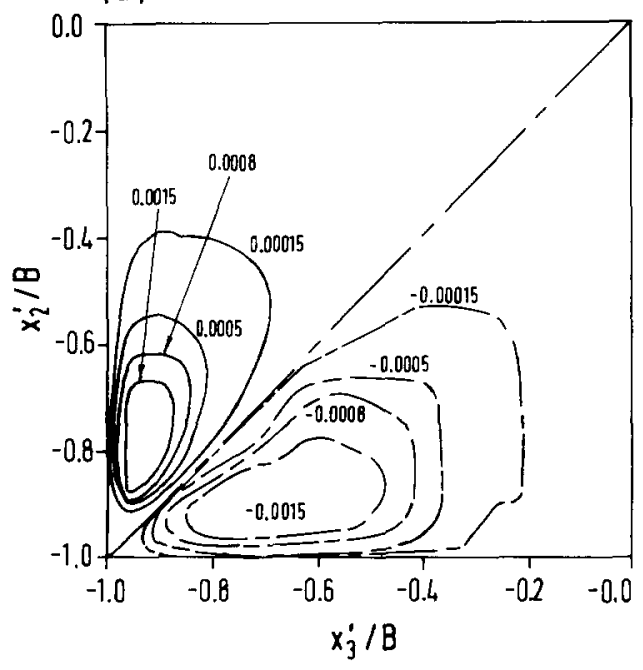

(c)

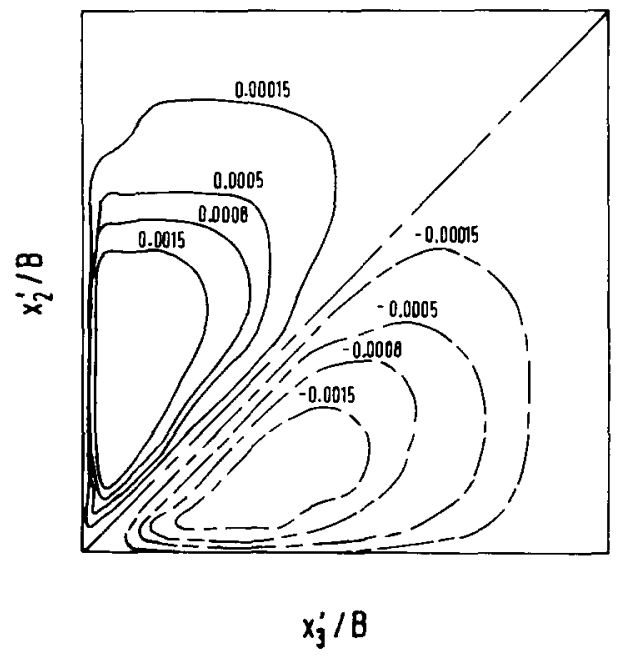

(b)

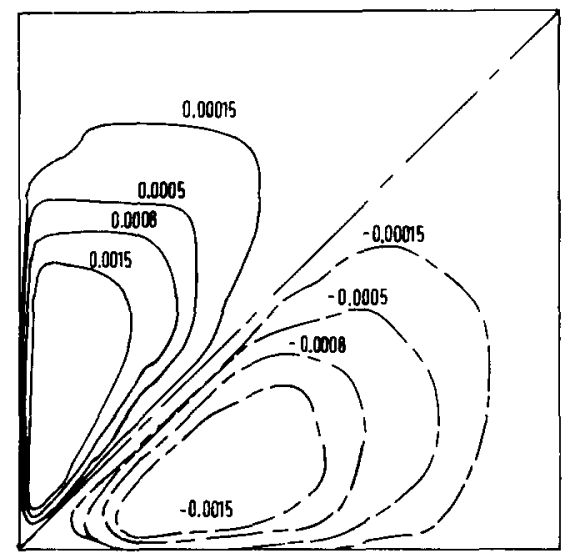


close to the fully developed flow condition may be assumed at $x_{1}^{\prime} / D \simeq 42$ for experimental measurements compatible with the accuracy of the measuring instrument. Generally, from magnitude of the $\psi$ contours, one can also deduce that a pair of counter-rotating vortices diffuses outwards from the corners with a corresponding shift of the vortex centers as the flow progresses downstream. The two turbulence models, TM I and TM II, produce fairly similar results especially for the fully developed state. At the lower downstream location, there are some differences in the contour quantities of $\psi$, but this may be attributed to the undue influence of the initial conditions specified which effect is not completely damped out by viscosity.

The secondary velocity profile for $R=250000$ is shown next in Fig. 5 where the shear velocity based on wall mean shear stress $\left(U_{t}\right)$ is used for non-dimensionalization purpose. Fig. 5(a) shows the distribution taken along the wall bisector while Fig. 5(b) shows the distribution where the corner bisector is the abscissa. In Fig. 5(a), there is significant underprediction among the various numerical models, including TM I, when compared to the experimental results of Gessner and Emery [7]. Only the model TM II seems to conform to the large value of $U_{2}^{\prime} / U_{\tau}$, and agreement with the experimental data is even better for the region further away from the wall. Although underpredicting, model TM I shows fairly similar behavior as the anisotropic $k-\varepsilon$ model of Myong and Kobayashi [17] and both are better off than the results of Demuren and Rodi 14] Fig. 5(b) shows similar feature of underprediction of $U_{2}^{\prime} / U_{t}$ by the different numerical models except for TM II, which shows the closest agreement to the experimental measurements of Gessner and Emery
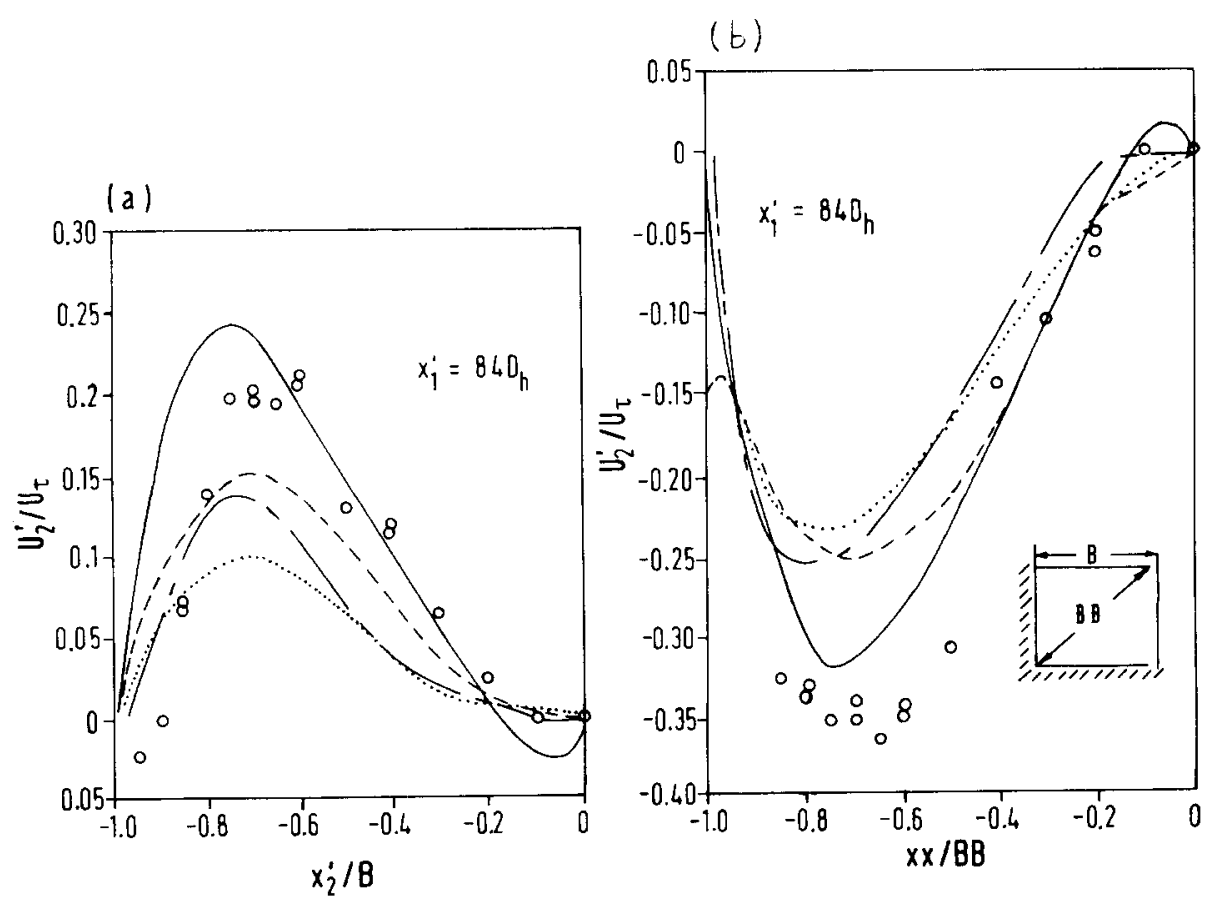

Fig. 5. Distribution of secondary velocity profiles $\left(U_{2}^{\prime} / U_{\tau}\right)$ at $R=250000$ for (a) wall bisector; (b) corner bisector. Present computations (- - ) TM I and (-) TM II; (- - ) computations by Myong and Kobayashi [17], (......) Demuren and Rodi [14]; ( $)$ experimental results by Gessner and Emery [7]. 
[7]. Generally, all the numerical models exhibit a consistent trend of increasing secondary velocity slightly away from the wall or corner, reaches a maximum and decreases towards the central region. This is consistent with the plot of Fig. 3 showing the bulging of the primary velocity contour towards the corner region and its associated larger magnitude of secondary flow quantities.

The fully-developed anisotropy of turbulence as indicated by the transverse Reynolds stress, $\left(\overline{u_{3}^{\prime} u_{3}^{\prime}}-\overline{u_{2}^{\prime} u_{2}^{\prime}}\right) / U_{1 \max }^{\prime 2}$, is plotted in Fig. 6. Fig. 6(a) shows the results obtained from TM I and TM II

(a)

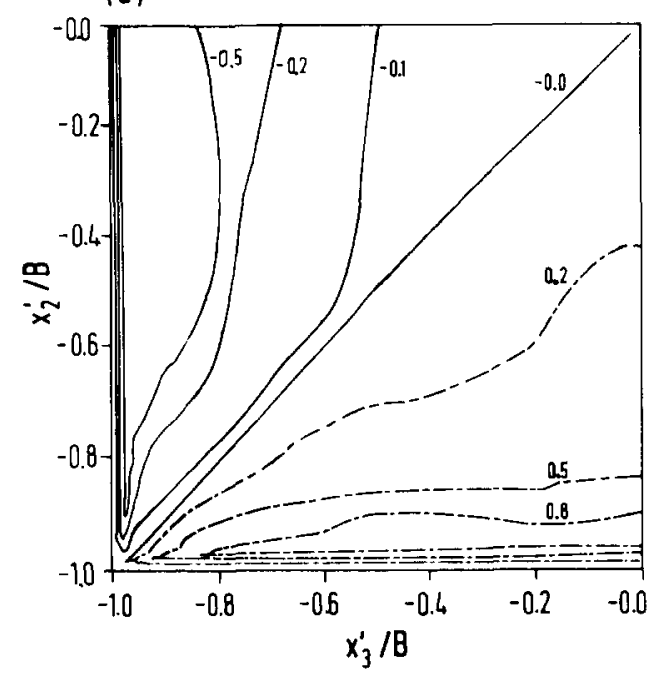

(c)

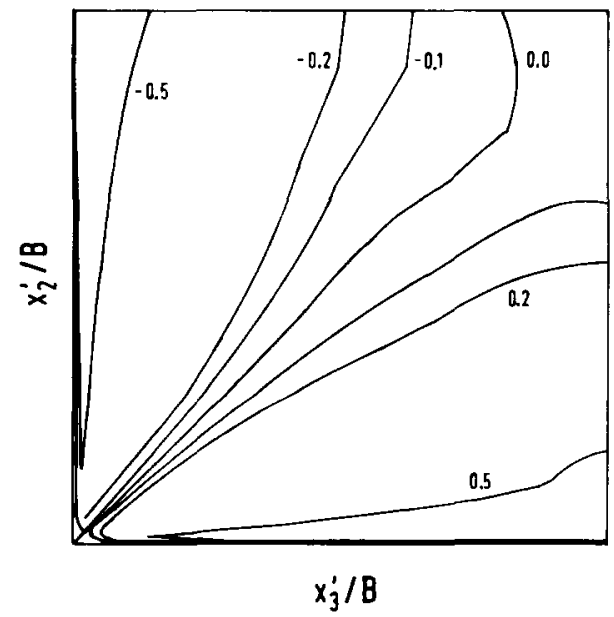

(b)

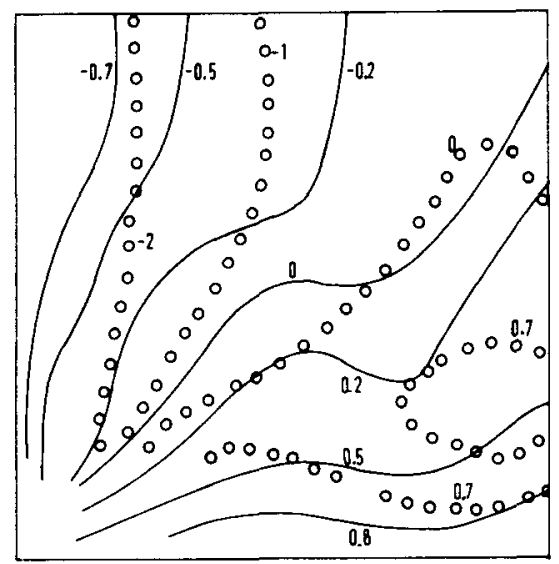


bearing reasonable agreement with each other. Better concurrence is found for the larger values of transverse stress close to the walls. Although the experimental measurements of Fujita et al. [9] in Fig. 6(b) indicates some asymmetry about the corner bisector, there is still general and fair agreement of trend when compared to the numerical prediction of Fig. 6(a). There is even more asymmetry of experimental results provided by Melling and Whitelaw [21] in the same Fig. 6(b), which may be indicative of the experimental difficulties of measuring such small quantities. Other numerical results by Nisizima [18] shown in Fig 6(c) using the anisotropic $k-\varepsilon$, turbulence model depict fairly symmetrical behavior and bear rather close resemblance to our results.

Contours of the fully developed lateral Reynolds stress, $\left(\overline{u_{1}^{\prime} u_{3}^{\prime}} / U_{1 \max }^{\prime 2}\right)$, are calculated for models TM II and TM I and shown in Fig. 7(a) and (b), respectively. Unlike the distribution in the

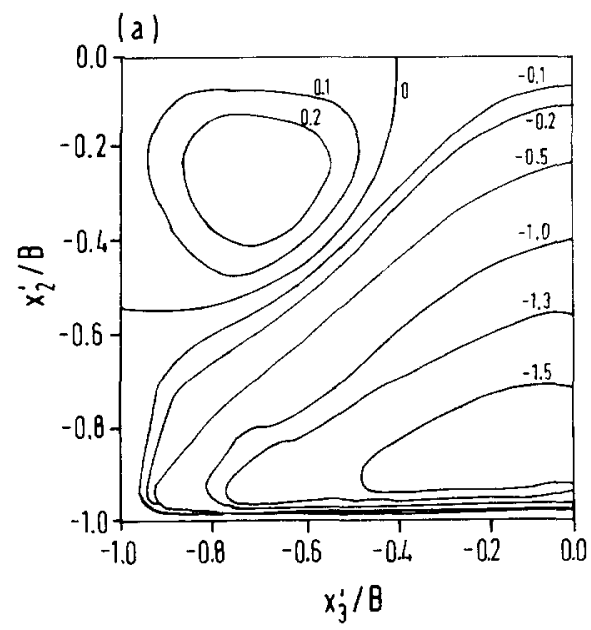

(b)

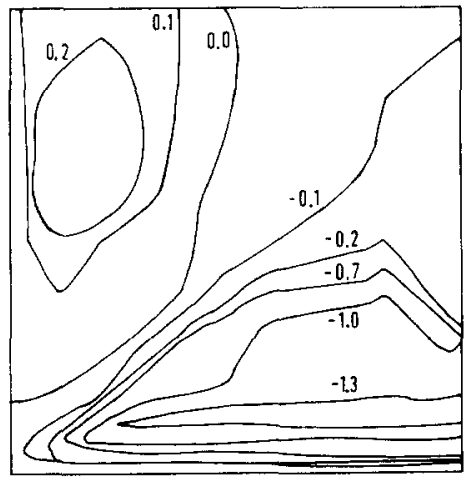

(c)

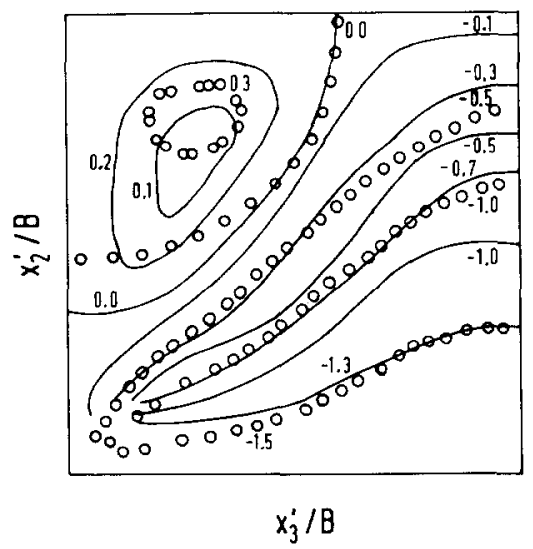

(d)

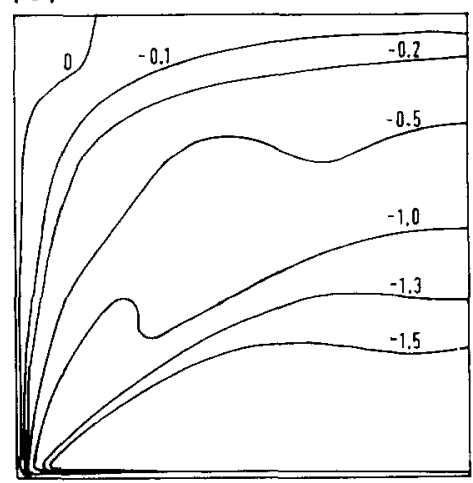

Fig. 7. Contours of lateral Reynolds stress $\left(\overline{u_{1}^{\prime} u_{3}^{\prime}} / U_{1 \max }^{2}\right) \times 10^{3}$ for (a) present computation at $R=65000(-) \mathrm{TM}$ II; (b) present computation at $R=65000$ (- - - ) TM I; (c) experimental results: ( $\longrightarrow$ ) Yokosawa et al. [22] at $R=65000$; (o) Melling and Whitelaw [21] at $R=42000$ for $(-1.5-1,-0.5,0,0.3)$; (d) computation by Nisizima [18] at $R=40806$. 
transverse Reynolds stress, the quantity $\left(\overline{u_{1}^{\prime} u_{3}^{\prime}} / U_{1 \max }^{\prime 2}\right)$ is only expected to be symmetrical about the wall bisector. Figs. 7(a) and (b) show broad agreement of results with higher magnitudes manifested closer to the walls. The experimental measurements by Yokosawa et al. [22] in Fig. 7(c) indicates general concurrence, with a marginally better agreement of magnitude for the case of model TM II. The conspicuous absence of data near one side of the wall, which also happens to the experimental results of Melling and Whitelaw [21], may be attributed to the difficulties of measurement and the strong wall effect on the accuracy of measurement. Our results when compared to the other numerical prediction of Nisizima [18] in Fig. 7(d) indicates better accord with the experimental data. The latter's computation of the closed contour quantities, if any, is much closer to the wall than that shown by the experimental results.

Fig. 8 compares the predicted and measured distributions of the turbulent kinetic energy along (a) the wall bisector and (b) the corner bisector for $R=250000$ at streamwise location of $x_{1}^{\prime} / D_{h}=84$. Generally, the models TM I and TM II show a consistent trend with the experimental results of Gessner and Emery [7], with a large magnitude closer to (a) the wall and (b) the corner
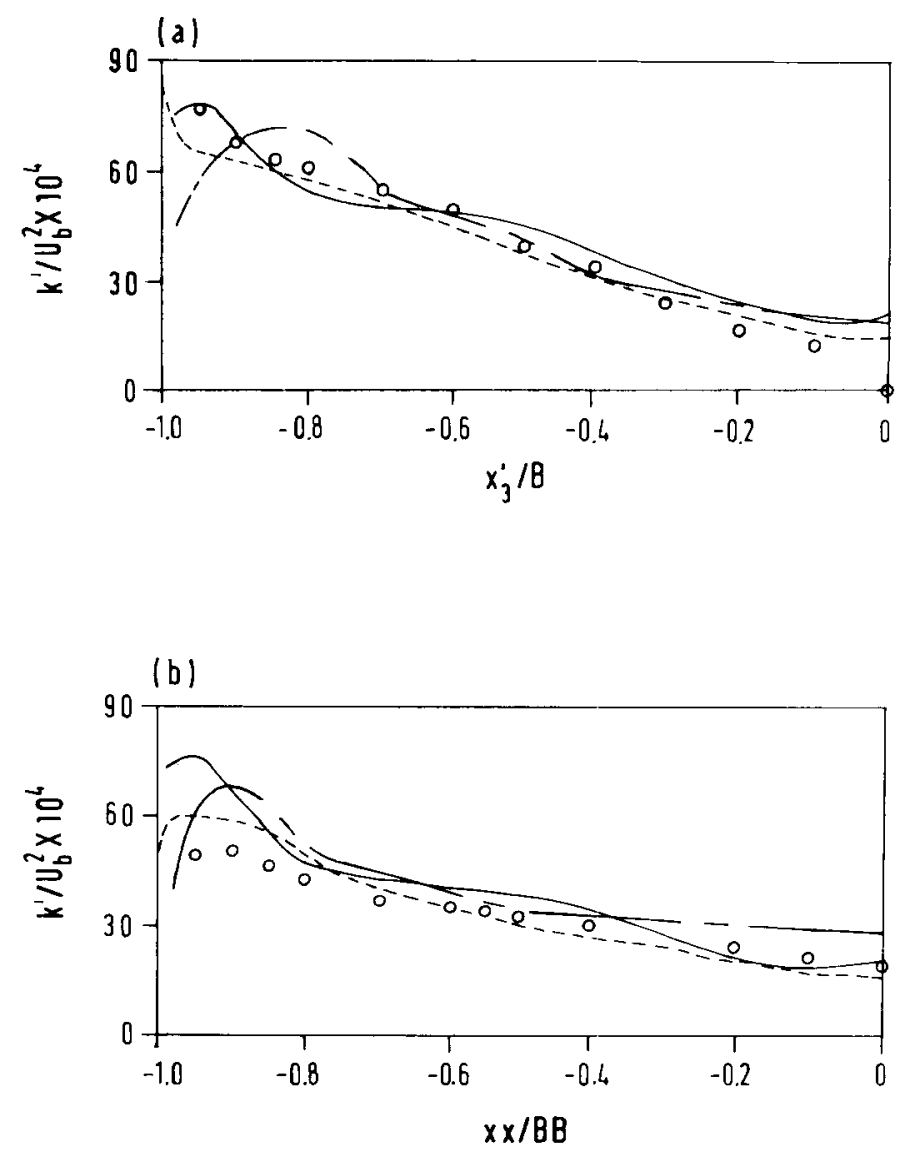

Fig. 8. Distribution of turbulent kinetic energy profiles at $R=25000$ for (a) wall bisector, (b) corner bisector. Present computation (- - - ) TM I and (_) TM II; (-- ) computations by Myong and Kobayashi [17]; (०) experimental results by Gessner and Emery [7]. 
and decreasing continuously towards the central region. This is also in agreement with the predicted results of Myong and Kobayasi [17] except that the latter showed a sharp peak near the wall (Fig. 8(a) while along the corner bisector, it does not possess this characteristic. This has been attributed by Myong and Kobayashi to the presence of strong secondary motion near the corner but the feature of the peak turbulent energy near the wall bisector is not quite apparent in either the exponential results or our computations.

Finally, the axial vorticity plot for $R=65000$ is shown in Fig. 9. Fig. 9(a), computed according to TM I, depicts the presence of two counter-rotating vortices in the flow which shows fair

(a)

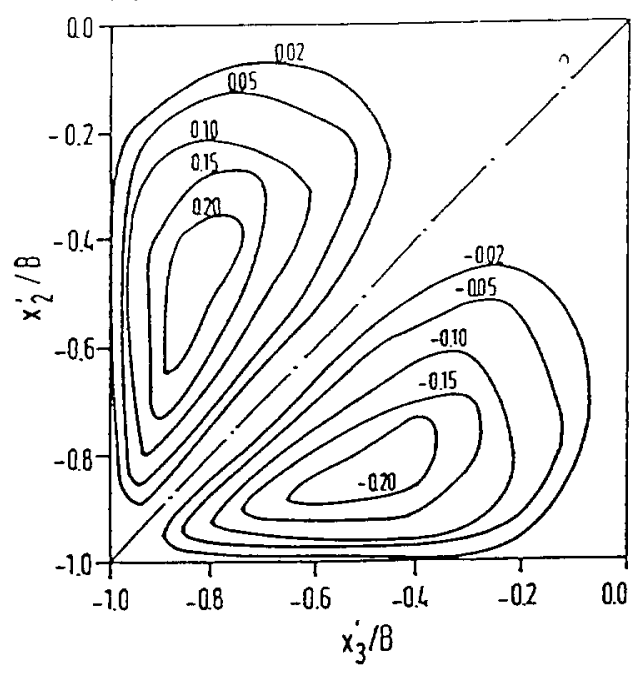

(c)

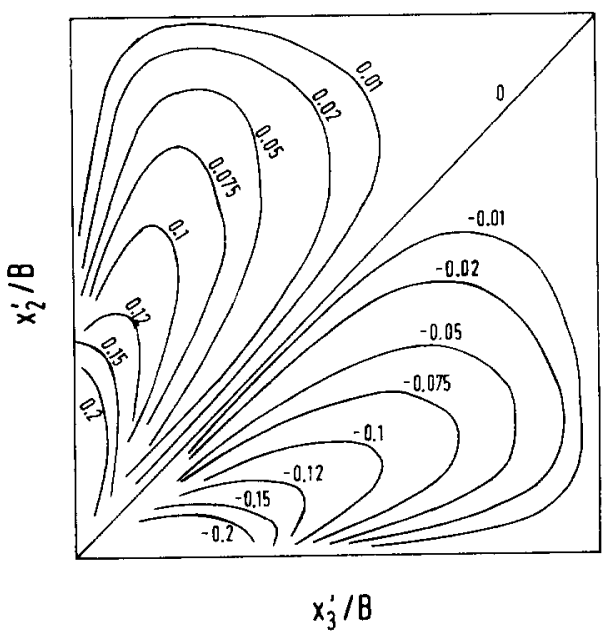

$\dot{x}_{3} / 8$ (b)

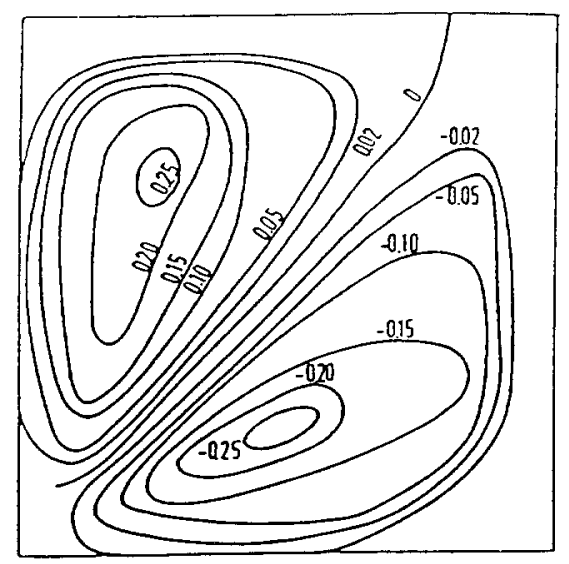


resemblance to the experimental results of Fujita et al. [9]. Considering the fact that the experimental results were obtained from numerically differentiating the measured secondary flow velocities, which are often of small magnitudes, the slight asymmetry detected in Fig. 9(b) is quite remarkable. Although Hoagland [2] managed to obtain a more symmetrical plot for the measured vorticity (Fig. 9(c)) which also shows comparable agreement, the magnitude of the contours is not as close to either our prediction or the results obtained by Fujita et al. Fig. 9(a) denotes that the peak vorticity is quite close to the wall in the respective quadrant.

\section{Concluding remarks}

A hybrid method is chosen for the numerical integration of the flow equations governing the turbulent flow in a square duct section. Since the governing equation is described in a multi-region framework in the cross-sectional plane, a high-order finite element technique is aptly utilized for computation to ensure $C^{1}$ continuity of solution and its higher-order derivatives across each region. It also greatly facilitates future work investigating the effect of flow through ducts of irregular and changing cross-sectional shapes as in most engineering applications. In the streamwise direction, a finite difference technique is employed for integration whereby subsequent (future) work involving changes from the present configuration of a straight duct to one with radius of curvature can be easily implemented.

In the present study of turbulent flow through a straight square duct, two different turbulence models are employed, namely, the two-layer model of Chen and Patel [15] and the isotropic $k-\varepsilon$ model with specified wall functions as described in [14]. Both models give comparable solutions and compare very well with previous experimental results and the recent numerical works employing anisotropic $k-\varepsilon$ turbulence modelling. Because the Chen and Patel model uses the wall boundary conditions as present in the physical flow, it provides us with further assurance for general application to other turbulent flows. On the other hand, the isotropic $k-\varepsilon$ model adopted can still be used for predicting purposes provided the wall function is specified accurately for the implementation of the wall boundary condition.

\section{Acknowledgement}

One of the authors (F.M. Wang) is sponsored by the Economic Development Board of Singapore. The computations were carried out on the University's IBM 3081 computer and the NEC SX-1A supercomputer.

\section{References}

[1] J. Nikuradse, "Turbulente Strömung in nicht-kreisförmigen rohren", Ing. Arch. 1, pp. 306-332, 1930.

[2] L.C. Hoagland, Fully developed turbulent flow in straight rectangular duct - secondary flow, its cause and effect on the primary flow, Ph.D. Thesis, Dept. Mech. Eng., M.I.T., Cambridge, 1960.

[3] E. Brundett and W.D. Baines, "The production and diffusion of vorticity in duct flow", J. Fluid Mech. 19, pp. 375-394, 1964. 
[4] F.B. Gessner and J.B. Jones, "On some aspects of fully developed turbulent flow in a rectangular channel", J. Fluid Mech. 23, pp. 689-713, 1965.

[5] H.J. Perkins, "The formation of streamwise vorticity in turbulent flow", J. Fluid Mech. 44, pp. 721 740, 1970.

[6] B.E. Launder and M.M. Ying, "Secondary flows in ducts of square cross-section", J. Fluid Mech. 54, pp. 289-295, 1972.

[7] F.B. Gessner and A.F. Emery, "The numerical prediction of developing turbulent flow in rectangular ducts". Trans. ASME J. Fluids Eng. 103, pp. 445-455, 1981.

[8] A.A.M. Aly, A.C. Trupp and A.D. Gerrard, "Measurements and prediction of fully developed turbulent flow in an equilateral triangular duct", J. Fluid Mech. 85, pp. 57-83, 1978.

[9] H. Fujita, H. Yokosawa and M. Hirota," Secondary flow of the second kind in rectangular ducts with one rough wall', Exp. Thermal Fluid Sci. 2, pp. 72-80, 1989.

[10] D.E. Metzger and R.P. Vedula, "Heat transfer in triangular channels with angled ribs on two walls", Exp. Heat Transfer 1, pp. 31-44, 1987.

[11] V. Ramachandra, The numerical prediction of flow and heat transfer in rod-bundle geometries, Ph.D. Thesis, University of London, 1979.

[12] D.D. Gosman and C.W. Rapley, "A prediction method for fully developed flow through non-circular passages", in: C. Taylor et al. (eds.) Numerical Method in Laminar and Turbulent Flows, 1978.

[13] S.V. Patankar and D.B. Spalding, "A calculation procedure for heat, mass and momentum transfer in threedimensional parabolic flows", Int. J. Heat Mass Transfer 15, pp. 1787-1806, 1972.

[14] A.O. Demuren and W. Rodi, "Calculation of turbulence-driven secondary motion in non-circular ducts", J. Fluid Mech. 140, pp. 189--222, 1984.

[15] H.C. Chen and V.C. Patel, "Practical near-wall turbulence models for complex flows including separation", AIAA J. 26, pp. 641-684, 1988.

[16] H.K. Myong, "Numerical investigation of fully developed turbulent flow and heat transfer in a square duct", Int. J. Heat Fluid Flow 12, pp. $344-351,1991$.

[17] H.K. Myong and T. Kobayashi, "Prediction of three-dimensional turbulent flow in a square duct with an anisotropic low Reynolds number $k-8$ model", Trans. ASME J. Fluids Eng. 113, pp. 608-615, 1991.

[18] S. Nisizima, "A numerical study of turbulent square-duct flow using an anisotropic $k \varepsilon$ model", Theoret. Comput. Fluid Dyn. 2(1), pp. 61-71, 1990.

[19] B.E. Launder, G. Reece and W. Rodi, "Progress in the development of Reynolds Stress turbulent closure", J. Fluid Mech. 68, pp. 537--568, 1975.

[20] J. Laufer, Investigation of turbulent flow in a two-dimensional channel, NASA Report 1053, 1951.

[21] A. Melling and J.H. Whitelaw, "Turbulent flow in a rectangular duct", J. Fluid. Mech. 78, pp. 289-315, 1976.

[22] H. Yokosawa, H. Fujita, M. Hirota and S. Iwata, "Measurement of turbulent flow in a square duct with roughened walls on two opposite sides", Int. J. Heat Fluid Flow 10, pp. 125 130. 1989. 